\title{
The effect of a 5-year hand hygiene initiative based on the WHO multimodal hand hygiene improvement strategy: an interrupted time-series study
}

Yumi Suzuki ${ }^{1,2^{*}}$ (D, Motoko Morino ${ }^{2,3}$, Ichizo Morita ${ }^{4}$ and Shigenori Yamamoto ${ }^{1}$

\begin{abstract}
Background: A World Health Organization (WHO) guideline-based multimodal hand hygiene $(\mathrm{HH})$ initiative was introduced hospital-wide to a nonteaching Japanese hospital for 5 years. The objective of this study was to assess the effect of this initiative in terms of changes in alcohol-based hand rub (ABHR) consumption and the Hand Hygiene Self-Assessment Framework (HHSAF) score.

Methods: The consumption of monthly hospital-wide ABHR was calculated in L per 1000 patient days (PDs). The change in ABHR consumption was analysed by an interrupted time series analysis with a pre-implementation period of 36 months and an implementation period of 60 months. The correlation between annual ABHR consumption and the HHSAF score was estimated using Pearson's correlation coefficients.

Results: The annual ABHR consumption was 4.0 (L/1000 PDs) to 4.4 in the pre-implementation period and 10.4 to 34.4 in the implementation period. The HHSAF score was 117.5 (out of 500) in the pre-implementation period and 267.5 to 445 in the implementation period. A statistically significant increase in the monthly ABHR consumption (change in slope: $+0.479 \mathrm{~L} / 1000 \mathrm{PDs}, p<0.01$ ) was observed with the implementation of the initiative. Annual ABHR consumption was strongly correlated with the annual HHSAF score $(r=0.971, p<0.01)$.

Conclusions: A 5-year WHO-based HH initiative significantly increased ABHR consumption. Our study suggested that the HHSAF assessment can be a good process measure to improve $\mathrm{HH}$ in a single facility, as ABHR consumption increased with the HHSAF score.
\end{abstract}

Keywords: Infection prevention, Hand hygiene, Alcohol-based hand rub, Compliance, Multimodal initiative, World Health Organization

\footnotetext{
* Correspondence: yumiatwork@me.com

'Department of Pediatrics, National Hospital Organization (NHO) Shimoshizu

National Hospital, 934-5 Shikawatashi, Yotsukaido 284-0003, Chiba, Japan

${ }^{2}$ Division of Infection Control, NHO Shimoshizu National Hospital,

Yotsukaidou, Japan

Full list of author information is available at the end of the article
}

C C The Author(s). 2020 Open Access This article is licensed under a Creative Commons Attribution 4.0 International License, which permits use, sharing, adaptation, distribution and reproduction in any medium or format, as long as you give appropriate credit to the original author(s) and the source, provide a link to the Creative Commons licence, and indicate if changes were made. The images or other third party material in this article are included in the article's Creative Commons licence, unless indicated otherwise in a credit line to the material. If material is not included in the article's Creative Commons licence and your intended use is not permitted by statutory regulation or exceeds the permitted use, you will need to obtain permission directly from the copyright holder. To view a copy of this licence, visit http://creativecommons.org/licenses/by/4.0/ The Creative Commons Public Domain Dedication waiver (http://creativecommons.org/publicdomain/zero/1.0/) applies to the data made available in this article, unless otherwise stated in a credit line to the data. 


\section{Background}

Hand hygiene $(\mathrm{HH})$ plays a key role in preventing hospitalacquired infections, as it prevents the spread of infectious organisms from patient to patient through the contamination of healthcare workers (HCWs)' hands [1-3]. The World Health Organization (WHO) published a multimodal strategy to improve hand hygiene compliance in 2009 [4], and improved $\mathrm{HH}$ has been reported from several areas following national and subnational $\mathrm{HH}$ campaigns based on the WHO guidelines [3, 5-7]. However, there have been no active national or sub-national initiatives in Japan, and a study on $\mathrm{HH}$ compliance in 4 Japanese teaching hospitals reported an overall compliance of 19\% [8]. Additionally, paediatric long-term care facilities have shown low $\mathrm{HH}$ compliance $[9,10]$. Infection control in such settings is considered challenging because patients with heavy medical needs, such as ventilators and tube feeding, also require therapeutic and social activities, resulting in frequent interactions with many HCWs and nonmedical staff [11].

The WHO multimodal $\mathrm{HH}$ strategy includes 3 main concepts: the "My 5 Moments for Hand Hygiene" approach, the "five strategy components", and the "step-wise approach" [12]. The "My 5 Moments for Hand Hygiene" approach highlights indications for moments for $\mathrm{HH}$. The "five strategy components" refer to the implementation of multiple actions to tackle different obstacles and behavioural barriers to improving $\mathrm{HH}$. These include Component 1: System Change, Component 2: Training and Education, Component 3: Evaluation and Feedback, Component 4: Reminders in the Workplace, Component 5: Institutional Safety Climate for Hand Hygiene. These five components stand for both the "five elements of the WHO multimodal Hand Hygiene Improvement Strategy" in the Guide to Implementation (GTI) [12], and the "five components of the Hand Hygiene Self-Assessment Framework (HHSAF) [13]". The "step-wise approach" helps to develop and plan the hand hygiene improvement programme over time based on a rational sequence of activities. This approach includes the following five steps: Step 1: Facility preparedness, Step 2: Baseline evaluation, Step 3: Implementation, Step 4: Follow-up evaluation, and Step 5: Ongoing planning and review cycle. As the original concept of the WHO strategy is intended to make improvements in $\mathrm{HH}$ sustainable, not to be used as a single campaign, the entire cycle is recommended to be repeated for 5 years [12]. Some previous studies have reported the results from adopting some parts of this strategy, the majority of which focused only on the "My 5 Moments for Hand Hygiene" approach and the "five strategy components" [14], while several focused on the "step-wise approach" $[15,16]$. In spite of the original recommendations in the guidelines, however, to date no studies have implemented both the "five strategy components" and the "step-wise approach" for 5 consecutive years.
The Hand Hygiene Self-Assessment Framework (HHSAF) [13] is a validated, systematic tool with which a situational analysis of hand hygiene promotion and practices in each health-care facility can be obtained [17]. The HHSAF acts as a diagnostic tool, identifying key issues requiring attention and improvement. Repeated use of the HHSAF also allows visualization of progress over time [13]. This tool has now been adopted in more than 90 countries [18]. We recorded our annual HHSAF scores for 5 years, which enabled us to visualize our progress over time and to analyse the correlation of the score with alcohol-based hand rub (ABHR) consumption, a surrogate marker for $\mathrm{HH}$ compliance.

We decided to introduce a $\mathrm{HH}$ initiative following the WHO $\mathrm{HH}$ strategy as a whole, including the "step-wise approach" and the HHSAF in our hospital, in which no systematic measures on $\mathrm{HH}$ had been implemented before. The aim of this study was to assess the effect of a hospitalwide WHO guideline-based $\mathrm{HH}$ initiative for 5 years. We also examined the correlation between the HHSAF score and ABHR consumption as an exploratory analysis.

\section{Methods \\ Study design and statistical methods}

An interrupted time series analysis was performed to examine the change in monthly ABHR consumption with the implementation of the $\mathrm{HH}$ initiative. The correlation between the HHSAF score and ABHR consumption was assessed using Pearson's correlation coefficients. The analyses were performed using IBM SPSS version 24 (IBM; Armonk, New York, USA).

\section{Data collection}

The HH initiative started in April 2014 and continued for 5 years until March 2019. The pre-implementation period was from April 2011 to March 2014 (36 months), and the implementation period was from April 2014 to March 2019 (60 months). Monthly and annual ABHR consumption (L per 1000 patient days; L/1000 PDs) was calculated by dividing the total amount of ABHR delivered from the pharmacy, hospital-wide, by the corresponding periods' patient census (patient hospitalization days). Monthly ABHR consumption and patient hospitalization days are shown in the Supplementary file. ABHR consumption data were collected from April 2014 to March 2019 each month in a prospective manner, and data from April 2011 to March 2014 were collected retrospectively.

The annual HHSAF score was calculated at the end of each fiscal year from 2013 to 2019. Scores for 2011 and 2012 were calculated retrospectively. The same infection control nurse (ICN) and infection control doctor (ICD), both in charge of the hospital-wide $\mathrm{HH}$ initiative, confirmed the scores together throughout the study period. 


\section{Setting}

The study was conducted in the 440-bed NHO Shimoshizu National Hospital, located in Chiba, Japan. This hospital is not a typical 'teaching hospital', except for some specialties that have limited teaching functions. Four wards, with a total of 200 beds, are mainly for secondary care (mean length of stay: 9.4 to 20.0 days). The proportion of private rooms is between 42.9 and $66.6 \%$. Five wards, with a total of 240 beds, provide long-term care mainly for patients with complex chronic medical conditions and require heavy medical care, such as mechanical ventilation and/or tube feeding (mean length of stay: 99.5 to 898.6 days). These wards are similar to paediatric long-term care facilities referenced in $[9,10]$. The proportion of private rooms is between 30.0 and $42.9 \%$. On average, over a hundred patients a day required ventilators (including non-invasive positive pressure ventilators used only overnight) during the study period, although there are no intensive care unit (ICU) beds in the hospital. Patients in the long-term care wards have direct contact with several recreational staff members every day, in addition to clinical staff for medical care. The link nurses (LNs: young nurses in charge of infection control within their ward) calculated their ideal $\mathrm{HH}$ events per PD for each ward based on a survey established by the ICN, referring to a previous study that used a nursing activity recording system [19]. Every year, all nurses in each ward recorded their nursing activities for 24 consecutive hours for more than 3 separate days. The LNs calculated the total number of $\mathrm{HH}$ opportunities required per PD by analysing the nursing workload data with the " 5 Moments" indications and then dividing this by the number of patients hospitalized. The average data for the 3 days, which was considered to be the usual workload for the ward, was used as the estimated ideal $\mathrm{HH}$ events per PD. The ideal number of $\mathrm{HH}$ events per PD estimated for each ward ranged from 15 to 25 in the acute care wards and from 40 to 60 in the long-term wards. The ideal hospital-wide $\mathrm{HH}$ events per PD was approximately 38 , obtained by taking the average of the values for all wards. As $1.3 \mathrm{ml}$ of $\mathrm{ABHR}$ is dispensed in each $\mathrm{HH}$ event, adequate hospital-wide ABHR consumption for our goal of $100 \%$ compliance was estimated to be approximately $50 \mathrm{~L} / 1000$ PDs. The amount of $1.3 \mathrm{ml}$ was determined by the product manufacturer through their own experiments $[20,21]$. At least one bottle of ABHR was placed at the entrance of each patient's room, each of which had up to a maximum of 4 beds, throughout the study period. In the pre-implementation period, some wards had posters with phrases such as "One Procedure, One Hand Wash", which had not been changed for years. The LNs checked hand washing procedures in training sessions that were held twice a year for each ward by rubbing a solution on the hands that glows under UV light. These sessions were the only regular $\mathrm{HH}$ training sessions conducted in the hospital. $\mathrm{HH}$ compliance was monitored by direct observation irregularly once to a few times a year, but the auditors had received only very limited training prior to the implementation of the initiative. No systematic $\mathrm{HH}$ initiatives had been implemented, and nobody had overviewed the whole $\mathrm{HH}$ improvement programme of the hospital before the study. The hospital has an infection control team (ICT) with 0.3 full-time equivalent (FTE) ICD throughout the study and 1.0 FTE ICN from January 2013 to the end of the study. All HCWs who have direct contact with the patients were included as participants of the study.

\section{Interventions}

We conducted an original 5-year hospital-wide $\mathrm{HH}$ initiative from April 2014 to March 2019, implementing activities from all five strategy components, with no incentives given to the participants. Our programme was based on the Guide to Implementation (GTI) [12], but some minor changes and local adaptations were made to match our hospital culture, as was recommended. In addition to translating the English tools, we prepared original posters and reminders with pictures hand drawn by the ICT and educational videos with the hospital staff as the actors, filmed in the actual hospital setting to enhance comprehension. The details of the activities implemented each year are shown in Table 1.

We repeated the five steps of the step-wise approach each fiscal year. In the GTI, the length of time recommended for each step is as follows: Step 1, 2 months; Steps 2 and 3, 3 months; and Steps 4 and 5, 2 months. In our original programme, we minimized the length of time for Steps 2 and 4 by applying the data from the previous year as the baseline and the annual data as the follow-up data. The outline of each step, determined according to the GTI, is summarized below; 201X stands for 2014 to 2018, and 201X + 1 stands for 2015 to 2019.

Step 1 (April to May 201X): Facility preparedness: The infection control committee (ICC: the highest decisionmaking body on infection control) decided upon and declared the hospital-wide implementation of the WHO $\mathrm{HH}$ strategy, the annual aim, and the hospital-wide target amount of ABHR consumption. The ICT was assigned as the main promotion team, and the infection control managers (ICMs: the field leaders on infection control) were assigned to work as the $\mathrm{HH}$ leaders (champions) for each department and ward.

Step 2 (May 201X): Baseline evaluation: The hospital-wide ABHR consumption from the previous year was applied as the baseline. At least one direct observation session for each ward was completed by the ICT within the month.

Step 3 (May 201X to March 201X + 1): Implementation: Tools and activities from all five strategy components were selected and adapted as described in Table 1. Hospital-wide activities were mainly led by the ICT, and 
Table 1 Details of the activities corresponding to the five strategy components implemented each year

\begin{tabular}{|c|c|c|c|c|c|c|}
\hline & Activities of the initiative & Year 1 & Year 2 & Year 3 & Year 4 & Year 5 \\
\hline \multirow[t]{4}{*}{ Component 1} & Distribution of personal shoulder bags for ABHR bottles & $\times$ & $\times$ & $\times$ & $\times$ & $\times$ \\
\hline & Automatic ABHR dispensers at the door of each room & & $\times$ & $\times$ & $\times$ & $\times$ \\
\hline & Forms of ABHR (gel, foam, aroma, etc.) & & $\times$ & $\times$ & $\times$ & $\times$ \\
\hline & Hand moisturizer & & & & $\times$ & $\times$ \\
\hline \multirow[t]{8}{*}{ Component 2} & $\mathrm{HH}$ leader training sessions & $\times$ & & & & \\
\hline & WHO HH guidelines and tool kits in all computers & $\times$ & $\times$ & $\times$ & $\times$ & $\times$ \\
\hline & Regular $\mathrm{HH}$ training sessions for all staff & $\times$ & $\times$ & $\times$ & & \\
\hline & Mandatory $\mathrm{HH}$ training sessions for all staff & & & & $\times$ & $\times$ \\
\hline & Original $\mathrm{HH}$ training video made by the ICT \& ICMs & & $\times$ & $\times$ & $\times$ & $\times$ \\
\hline & E-learning using the original training video & & & $\times$ & $\times$ & $\times$ \\
\hline & On-the-job direct observation training for ICMs \& LNs & & & $x$ & $\times$ & $x$ \\
\hline & Hands on training sessions & & & & & $\times$ \\
\hline \multirow[t]{9}{*}{ Component 3} & ABHR consumption monitoring by the ICT (monthly) & $\times$ & $\times$ & $\times$ & $\times$ & $x$ \\
\hline & ABHR consumption monitoring by LNs of each ward & & $x$ & $\times$ & $\times$ & $\times$ \\
\hline & Personal ABHR consumption monitoring by LNs & & & & $\times$ & $\times$ \\
\hline & HH events per day survey by LNs & $\times$ & $\times$ & $\times$ & $\times$ & $\times$ \\
\hline & Direct observation by the ICT (twice a year per ward) & $\times$ & $\times$ & $x$ & $\times$ & $\times$ \\
\hline & Direct observation by LNs & & & $\times$ & $\times$ & $\times$ \\
\hline & Perception survey for senior executive managers & $\times$ & & & & \\
\hline & Perception survey for all staff & $\times$ & & & $\times$ & \\
\hline & Knowledge survey for all staff & $\times$ & & $\times$ & & \\
\hline \multirow[t]{6}{*}{ Component 4} & 5 Moments reminders on $A B H R$ bottles & $\times$ & $\times$ & $\times$ & $\times$ & $\times$ \\
\hline & $\mathrm{HH}$ procedure posters & $\times$ & $\times$ & $\times$ & $\times$ & $\times$ \\
\hline & Posters for each HH campaign & & $\times$ & $\times$ & $\times$ & $\times$ \\
\hline & 5 Moments posters with hand drawn pictures & & & $\times$ & $\times$ & $\times$ \\
\hline & Hand rub procedure reminders with hand drawn pictures & & & & & $\times$ \\
\hline & Reminders made by LNs for each ward & & & & & $\times$ \\
\hline \multirow[t]{11}{*}{ Component 5} & Letter to the director & $\times$ & & & & \\
\hline & Letters to the head of each department and ward & $\times$ & & & & \\
\hline & Assignment of ICMs as $\mathrm{HH}$ champions & $\times$ & $x$ & $x$ & $\times$ & $x$ \\
\hline & Selection of "HH masters" as role models & & & $x$ & $\times$ & $\times$ \\
\hline & HH campaign twice a year (May \& October) & $\times$ & $x$ & $\times$ & $\times$ & $\times$ \\
\hline & Campaign poster with a picture of the director & & & $\times$ & $\times$ & $\times$ \\
\hline & Campaign poster with a picture of the staff members & & & & $\times$ & $x$ \\
\hline & Institutional target & $\times$ & $x$ & $x$ & $\times$ & $x$ \\
\hline & Presentation sessions to share activities on $\mathrm{HH}$ & & & $\times$ & $\times$ & $\times$ \\
\hline & Newsletters with issues on $\mathrm{HH}$ & & & $\times$ & $\times$ & $\times$ \\
\hline & Inclusion of $\mathrm{HH}$ as part of the buddy training system & & & & & $\times$ \\
\hline
\end{tabular}

ABHR alcohol-based hand rub, Component 1 System Change, Component 2 Training and Education, Component 3 Evaluation and Feedback, Component 4 Reminders in the Workplace, Component 5 Institutional Safety Climate for Hand Hygiene, HH hand hygiene, ICT infection control team, ICM infection control manager, $L N$ link nurse

local activities were mainly led by the ICMs. LNs worked with the ICMs in the nursing departments.

Step 4 (March to April 201X +1 ): Follow-up evaluation: The ICT reported the annual (April 201X to
March $201 \mathrm{X}+1$ ) ABHR consumption, the findings from the direct observations, and the annual HHSAF score.

Step 5 (March to April 201X + 1): Ongoing planning and review cycle: The achievement of the year and the 
remaining challenges were evaluated by the ICC. The institutional aim, the target amount, and the target activities for the next year were planned by the ICT for continuous improvement.

\section{Results}

\section{Participants}

A total of $402 \mathrm{HCWs}$ worked full time in the hospital at the final month of the study and were all included as participants. This group comprised 37 physicians, 261 members of the nursing staff, 20 rehabilitation therapists, and 20 members of the recreational staff. The number of workers varied within the study period but showed no increasing or decreasing tendencies. The average number of monthly patient hospitalization days throughout the study period was 9970 . The average was 10,482 for the pre-implementation period and 9662.9 for the implementation period.

\section{Implementation of the initiative}

Table 2 shows the details of the five steps of the stepwise approach each year. For the first 2 years, most

Table 2 Details of the five steps of the step-wise approach in the 5-year cycle

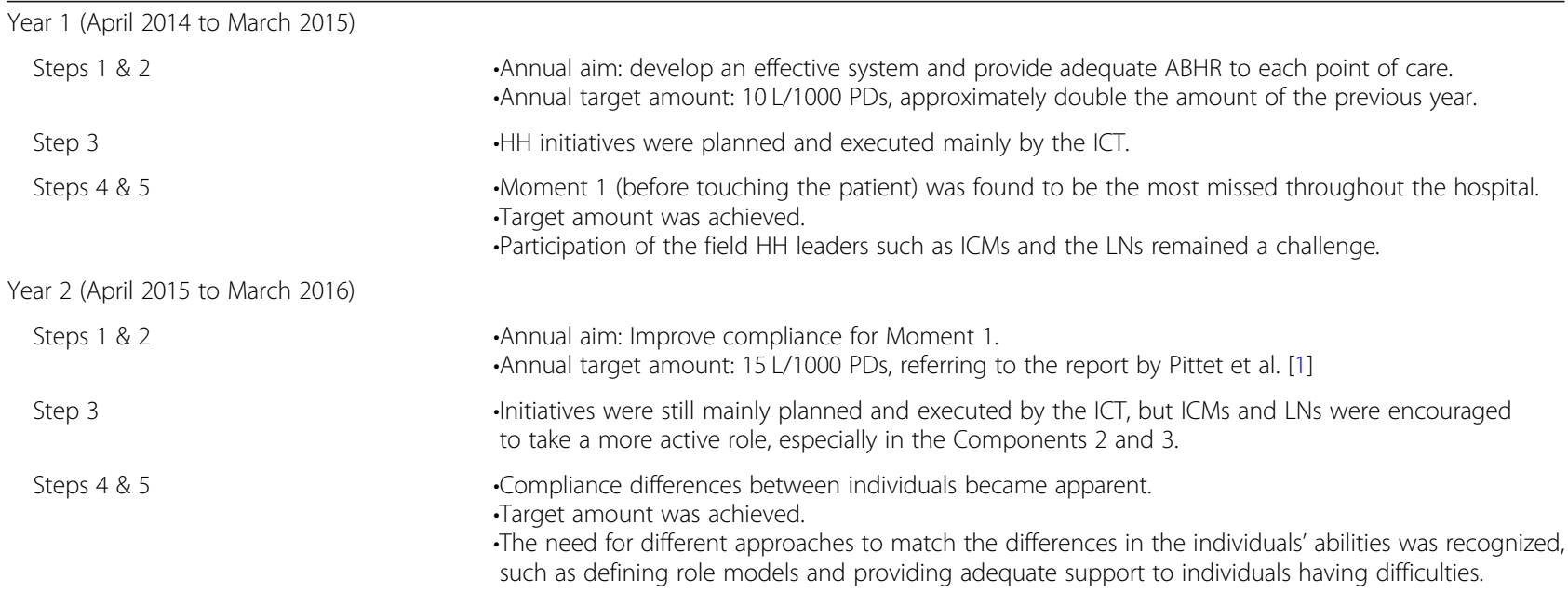

-Annual aim: Encourage individual support for staff with low compliance and promote the activities of the staff with high compliance, at each local field level. -Annual target amount: 25 L/1000 PDs, 1/2 the estimated adequate ABHR consumption.

-Many tools from Component 5 were utilized to reinforce field-based initiatives.

-The compliance differences between the wards and departments became apparent.

-Target amount was $91.6 \%$ achieved.

-Field-level HH initiatives of fields with high compliances should be shared.

-Annual aim: Share effective initiatives between wards and departments, focusing on Moment 1 again. This moment was selected as it was a common moment for every HCW, and sharing was expected to be effective.

-Annual target amount: 30 L/1000 PDs, 3/5 the estimated adequate ABHR consumption.

-Effective activities were shared in ICM meetings. The ICT provided 4 weeks of intensive support to several wards experiencing difficulties.

- HH was found to be missed in certain routine procedures, which differed between fields.

- Target amount was 99\% achieved.

-Voluntary activities of the ICMs and LNs should be further encouraged.

-Annual aim: Focus on $\mathrm{HH}$ in the routine work of each ward and department. -Annual target amount: 33 L/1000 PDs, 2/3 the estimated adequate ABHR consumption.

-ICMs and LNs reviewed and focused on the HH moment that tended to be missed in their everyday routine work procedures.

-Target amount was achieved.

-HHSAF assessment showed that Component 5 had the most room for improvement. 
activities were planned and conducted directly by the ICT. In the following years, plans were made so that the ownership of the $\mathrm{HH}$ initiatives would be transferred to the ICMs, the front-line champions, to let them lead local activities.

Table 3 shows the annual ABHR consumption and HHSAF score. The annual ABHR consumption ranged from 4.0 (L/1000 PDs) to 4.4 in the pre-implementation period and from 10.4 to 34.4 in the implementation period. The HHSAF score was 117.5 (out of 500) in the pre-implementation period and 267.5 to 445 in the implementation period. The HHSAF included another component; the "Leadership Criteria" for facilities that had reached the advanced level (score 376-500) of $\mathrm{HH}$, but this was not assessed during this study period.

Table 4 shows the details of the HHSAF score.

\section{Monthly ABHR consumption}

Figure 1 shows the trend of monthly ABHR consumption. The $r$ value for the regression model was 0.958 , and the adjusted $r^{2}$ value was 0.916 . The baseline ABHR consumption in the pre-implementation period was estimated to be $4.3 \mathrm{~L} / 1000$ PDs. This was stable with no observable trend in the baseline segment. At the start of the implementation of the hospital-wide $\mathrm{HH}$ initiative in April 2014, an immediate increase of 4.4 L/1000 PDs, approximately doubling the baseline ABHR consumption, was observed. Furthermore, there was a significant slope change of +0.479 ( $95 \%$ confidence interval: 0.359 to $0.599, p<0.001) \mathrm{L} / 1000$ PDs in the ABHR consumption before and after the implementation of the initiative. Table 5 summarizes the estimates from the segmented regression model.

\section{Annual ABHR consumption and HHSAF scores}

Table 3 shows the annual ABHR consumption and HHSAF score. Figure 2 shows that a significant positive correlation $(r=0.971, p<0.001)$ was found between the annual ABHR consumption and HHSAF score.

\section{Discussion}

A 5-year WHO-based $\mathrm{HH}$ initiative significantly increased ABHR consumption in a nonteaching secondary hospital in Japan with long-term care wards. In addition, HHSAF scores were also increased with a significant positive correlation with ABHR consumption.

The WHO guidelines recommend repeating the entire cycle of the step-wise approach for a minimum of 5 years. Despite the recommendation, the majority of previous studies reporting the effect of a multimodal WHO-based $\mathrm{HH}$ programme focused only on the five strategy components. Although several studies followed the step-wise programme $[15,16]$ or a 5-year programme $[22,23]$, to the best of our knowledge, there have been no previous studies reporting a 5-year programme with five cycles of the step-wise approach. Our 5-year initiative resulted in an eight-fold increase in ABHR consumption, from 4.2 to $34.4 \mathrm{~L} / 1000$ PDs, which is similar to the increase reported by Pittet, from 4.1 to $30.6 \mathrm{~L} / 1000$ PDs, over 7 years [24]. Additionally, our study is the first to report the 5-year change in the HHSAF score in a single facility, which showed a nearly four-fold increase, from 117.5 to 445 points.

The rationale for the importance of a 5-year programme is not given in the WHO guidelines. The target amount of ABHR consumption recommended in the guidelines is $20 \mathrm{~L} / 1000$ PDs (HHSAF: 3.3c), and in Pittet's study, published prior to the publication of the guidelines, a period of 5 years was necessary to increase ABHR consumption from 4.1 to 20.8 L/1000 PDs [24]. Additionally, previous studies have suggested that behavioural changes are important in the improvement of $\mathrm{HH}$ [25-27] and that this improvement took time, sometimes years. Similar results were reported from a 6-year initiative in a tertiary teaching hospital [28] and a 4-year initiative in a paediatric long-term care facility [29].

In our initiative, we put effort into increasing local field staff involvement each year, as our long-term patients had frequent daily contact with staff members from many different departments, and the effective

Table 3 Annual ABHR consumption and HHSAF score in each year

\begin{tabular}{llll}
\hline & Intervention Year & Annual ABHR Consumption (L/1000 PDs) & HHSAF Score (/500) \\
\hline Pre-implementation & Year -3 (Apr-11 to Mar-12) & 4.4 & 117.5 \\
& Year -2 (Apr-12 to Mar-13) & 4.0 & 117.5 \\
& Year -1 (Apr-13 to Mar-14) & 4.2 & 117.5 \\
Implementation & Year 1 (Apr-14 to Mar-15) & 10.4 & 267.5 \\
& Year 2 (Apr-15 to Mar-16) & 17.7 & 310.0 \\
& Year 3 (Apr-16 to Mar-17) & 22.9 & 380.0 \\
& Year 4 (Apr-17 to Mar-18) & 29.6 & 410.0 \\
& Year 5 (Apr-11 to Mar-19) & 34.4 & 445.0 \\
\hline
\end{tabular}


Table 4 Details of the Hand Hygiene Self-Assessment Framework Score

\begin{tabular}{|c|c|c|c|c|c|c|c|c|c|}
\hline & & Year -3 & Year -2 & Year -1 & Year 1 & Year 2 & Year 3 & Year 4 & Year 5 \\
\hline \multirow{8}{*}{ Component 1} & 1.1 & 10 & 10 & 10 & 30 & 50 & 50 & 50 & 50 \\
\hline & 1.2 & 5 & 5 & 5 & 5 & 5 & 5 & 5 & 5 \\
\hline & 1.3 & 10 & 10 & 10 & 10 & 10 & 10 & 10 & 10 \\
\hline & 1.4 & 10 & 10 & 10 & 10 & 10 & 10 & 10 & 10 \\
\hline & 1.5 & 10 & 10 & 10 & 10 & 10 & 10 & 10 & 10 \\
\hline & 1.6 & 10 & 10 & 10 & 10 & 10 & 10 & 10 & 10 \\
\hline & add & 0 & 0 & 0 & 0 & 0 & 0 & 0 & 0 \\
\hline & subtotal & 55 & 55 & 55 & 75 & 95 & 95 & 95 & 95 \\
\hline \multirow{10}{*}{ Component 2} & $2.1 a$ & 10 & 10 & 10 & 10 & 10 & 10 & 20 & 20 \\
\hline & $2.1 \mathrm{~b}$ & 20 & 20 & 20 & 20 & 20 & 20 & 20 & 20 \\
\hline & $2.2 \mathrm{a}$ & 0 & 0 & 0 & 5 & 5 & 5 & 5 & 5 \\
\hline & $2.2 b$ & 0 & 0 & 0 & 5 & 5 & 5 & 5 & 5 \\
\hline & $2.2 \mathrm{c}$ & 0 & 0 & 0 & 5 & 5 & 5 & 5 & 5 \\
\hline & $2.2 d$ & 0 & 0 & 0 & 5 & 5 & 5 & 5 & 5 \\
\hline & 2.3 & 0 & 0 & 0 & 0 & 0 & 15 & 15 & 15 \\
\hline & 2.4 & 0 & 0 & 0 & 0 & 0 & 0 & 0 & 15 \\
\hline & 2.5 & 0 & 0 & 0 & 0 & 0 & 0 & 10 & 10 \\
\hline & subtotal & 30 & 30 & 30 & 50 & 50 & 65 & 85 & 100 \\
\hline \multirow[t]{12}{*}{ Component 3} & 3.1 & 0 & 0 & 0 & 0 & 0 & 0 & 10 & 10 \\
\hline & $3.2 \mathrm{a}$ & 0 & 0 & 0 & 0 & 0 & 5 & 5 & 0 \\
\hline & $3.2 \mathrm{~b}$ & 5 & 5 & 5 & 5 & 5 & 5 & 0 & 5 \\
\hline & $3.3 \mathrm{a}$ & 0 & 0 & 0 & 5 & 5 & 5 & 5 & 5 \\
\hline & $3.3 b$ & 0 & 0 & 0 & 0 & 0 & 0 & 0 & 0 \\
\hline & $3.3 c$ & 0 & 0 & 0 & 0 & 0 & 5 & 5 & 5 \\
\hline & $3.4 a$ & 5 & 5 & 5 & 10 & 10 & 10 & 10 & 10 \\
\hline & $3.4 b$ & 0 & 0 & 0 & 15 & 20 & 20 & 20 & 20 \\
\hline & $3.5 a$ & 0 & 0 & 0 & 5 & 5 & 5 & 5 & 5 \\
\hline & $3.5 \mathrm{bi}$ & 0 & 0 & 0 & 0 & 7.5 & 7.5 & 7.5 & 7.5 \\
\hline & 3.5bii & 0 & 0 & 0 & 0 & 7.5 & 7.5 & 7.5 & 7.5 \\
\hline & subtotal & 10 & 10 & 10 & 40 & 60 & 70 & 75 & 75 \\
\hline \multirow[t]{8}{*}{ Component 4} & $4.1 a$ & 0 & 0 & 0 & 20 & 20 & 20 & 25 & 25 \\
\hline & $4.1 \mathrm{~b}$ & 5 & 5 & 5 & 5 & 5 & 5 & 5 & 15 \\
\hline & $4.1 \mathrm{c}$ & 7.5 & 7.5 & 7.5 & 7.5 & 10 & 10 & 10 & 10 \\
\hline & 4.2 & 0 & 0 & 0 & 0 & 0 & 10 & 10 & 10 \\
\hline & 4.3 & 0 & 0 & 0 & 10 & 10 & 10 & 10 & 10 \\
\hline & 4.4 & 0 & 0 & 0 & 0 & 0 & 10 & 10 & 10 \\
\hline & 4.5 & 0 & 0 & 0 & 15 & 15 & 15 & 15 & 15 \\
\hline & subtotal & 12.5 & 12.5 & 12.5 & 57.5 & 60 & 80 & 85 & 95 \\
\hline \multirow[t]{6}{*}{ Component 5} & $5.1 a$ & 5 & 5 & 5 & 5 & 5 & 5 & 5 & 5 \\
\hline & $5.1 b$ & 5 & 5 & 5 & 5 & 5 & 5 & 5 & 5 \\
\hline & $5.1 \mathrm{c}$ & 0 & 0 & 0 & 0 & 0 & 5 & 5 & 5 \\
\hline & $5.2 a$ & 0 & 0 & 0 & 10 & 10 & 10 & 10 & 10 \\
\hline & $5.2 b$ & 0 & 0 & 0 & 0 & 0 & 0 & 0 & 0 \\
\hline & $5.2 \mathrm{C}$ & 0 & 0 & 0 & 5 & 5 & 5 & 5 & 5 \\
\hline
\end{tabular}


Table 4 Details of the Hand Hygiene Self-Assessment Framework Score (Continued)

\begin{tabular}{|c|c|c|c|c|c|c|c|c|c|}
\hline & & Year -3 & Year -2 & Year -1 & Year 1 & Year 2 & Year 3 & Year 4 & Year 5 \\
\hline & 5.3 & 0 & 0 & 0 & 10 & 10 & 10 & 10 & 10 \\
\hline & $5.4 a$ & 0 & 0 & 0 & 5 & 5 & 5 & 5 & 5 \\
\hline & $5.4 \mathrm{~b}$ & 0 & 0 & 0 & 0 & 0 & 5 & 5 & 5 \\
\hline & $5.5 a$ & 0 & 0 & 0 & 0 & 0 & 0 & 0 & 0 \\
\hline & $5.5 b$ & 0 & 0 & 0 & 0 & 0 & 0 & 0 & 0 \\
\hline & $5.6 a$ & 0 & 0 & 0 & 0 & 0 & 5 & 5 & 5 \\
\hline & $5.6 b$ & 0 & 0 & 0 & 5 & 5 & 5 & 5 & 5 \\
\hline & $5.6 \mathrm{c}$ & 0 & 0 & 0 & 0 & 0 & 5 & 5 & 5 \\
\hline & $5.6 d$ & 0 & 0 & 0 & 0 & 0 & 5 & 5 & 5 \\
\hline & $5.6 e$ & 0 & 0 & 0 & 0 & 0 & 0 & 0 & 5 \\
\hline & $5.6 f$ & 0 & 0 & 0 & 0 & 0 & 0 & 0 & 5 \\
\hline & subtotal & 10 & 10 & 10 & 45 & 45 & 70 & 70 & 80 \\
\hline total HHSAF score & & 117.5 & 117.5 & 117.5 & 267.5 & 310 & 380 & 410 & 445 \\
\hline
\end{tabular}

Component 1 System Change, Component 2 Training and Education, Component 3 Evaluation and Feedback, Component 4 Reminders in the Workplace, Component 5 Institutional Safety Climate for Hand Hygiene, HHSAF Hand Hygiene Self-Assessment Framework

measures differed between them. During the first 3 years, the ICT began implementing new systems for the ICMs to work as active field $\mathrm{HH}$ leaders. Then, in the last 2 years, the ICMs led the "local, focused implementation" of the $\mathrm{HH}$ initiative with the support of the LNs. From these experiences, we retrospectively recognized that 5 years was a reasonable length of time to change systems and to embed frontline staff engagement for local focused implementation.

Our annual hospital-wide $\mathrm{HH}$ compliance, monitored by direct observation, nearly tripled in the first 2 years after initiation of the intervention, which then ranged from 68 to $70 \%$ from the second year to the fifth year. We continuously conducted direct observations, but we

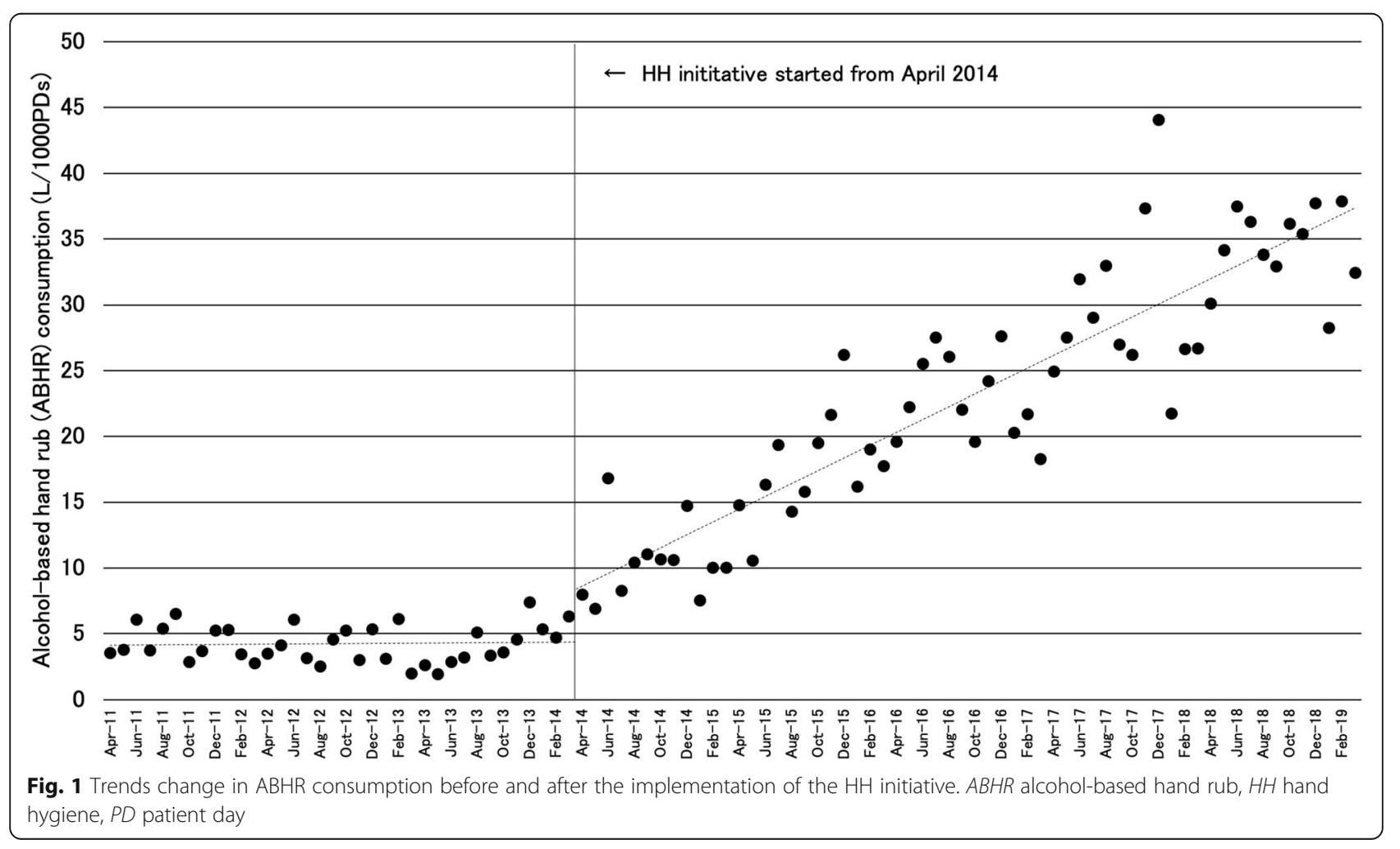


Table 5 Parameter estimates, 95\% Cls and P-values from segmented regression model describing the trends of monthly ABHR consumption

\begin{tabular}{|c|c|c|c|}
\hline & Coefficient & $95 \% \mathrm{Cl}$ & $P$-value \\
\hline Intercept & 4.344 & $2.032-6.656$ & $<0.001$ \\
\hline Baseline trend & 0.006 & $-0.103-0.115$ & 0.919 \\
\hline Level change from last point in the pre-implementation to the first point in the implementation phase & 4.387 & $1.499-7.276$ & 0.003 \\
\hline Slope change from pre-implementation to implementation & 0.479 & $0.359-0.599$ & $<0.001$ \\
\hline
\end{tabular}

$A B H R$ alcohol-based hand rub, $\mathrm{Cl}$ confidence interval

The model describes trends and change in the error rate during the pre-implementation, and implementation phases

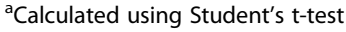

did not include any of these data as an outcome or quantitative measure, as we were unable to regard our quantitative data as reliable for several reasons. First, prior to the implementation of the initiative, there was a lack of trained auditors who could conduct the direct observations accurately. Second, because our initiative was carried out with no additional staff reinforcement or high-tech recording devices, only overt observations were possible. We observed the "Hawthorn effect" in most of the direct observations, which became more obvious in the latter phase of the initiative. In the final years of the intervention, the behaviours of the HCWs began to change within minutes of the start of each observation session. Third, there was also a large 'observer bias', as the auditors tended to follow HCWs with low compliances to determine the main obstacles for improving $\mathrm{HH}$ compliance.

On the other hand, we were able to obtain much informative qualitative data by "examining in detail the

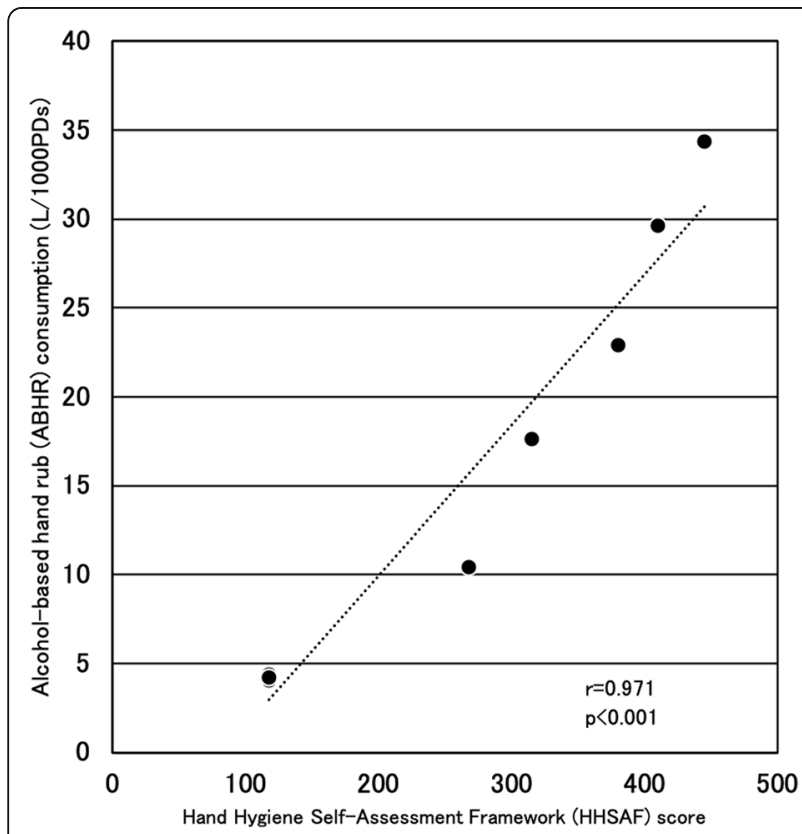

Fig. 2 Relationship between annual ABHR consumption and HHSAF score. ABHR alcohol-based hand rub, HHSAF Hand Hygiene SelfAssessment Framework, PD patient day barriers and opportunities to increase $\mathrm{HH}$ compliance" [30]. Therefore, rather than emphasizing the quantitative data, we focused on showing where improvement was most required specifically for the field staff, preparing effective training programmes for the following year, and determining our annual institutional aim based on the findings from the direct observations. For example, the annual aim chosen for the second year was to "Improve compliance for Moment 1 (before touching the patient)", as this was found to be the most missed of the " $5 \mathrm{Mo}$ ments" throughout the hospital during the previous year.

The current gold standard for $\mathrm{HH}$ compliance monitoring is unobtrusive direct observation [31]. However, conducting unobtrusive observations continuously and daily for years is difficult for most hospitals and cannot be widely recommended in terms of feasibility. High-tech recording devices are available, but most nonteaching hospitals in Japan cannot afford them. It is also said that direct observations can only catch a very small proportion of the actual $\mathrm{HH}$ events performed [32]. ABHR consumption, a surrogate marker for monitoring $\mathrm{HH}$ compliance, can be monitored easily and continuously for years and can also give a 24-h picture of compliance for all clinicians [33]. ABHR consumption monitoring has been officially recommended in "The surveillance procedures for small and medium sized medical facilities" since 2009 in Japan and has also been applied by the European Centre for Disease Prevention and Control [34] for standardized surveillance purposes. Many reports from Europe [35-38] and a report from Africa [39] have indicated the adoption of indirect monitoring of $\mathrm{HH}$ activity based on an ABHR consumption system. As long as direct observations are also conducted for qualitative measures and no punitive approaches are taken, ABHR consumption monitoring may be considered as a practical measure, especially for assessing improvement in long-term initiatives, for facilities with limited resources.

The WHO guidelines state that Step 5 of the stepwise approach is a crucial step for developing long-term plans to ensure that improvement is sustained and progresses. Reviewing our present position with ABHR consumption, findings from the direct observations, and the HHSAF score each year provided us with a bird's eye view of what 
we have accomplished and what is left to be done. The HHSAF helps to identify key issues requiring attention and the resources and tools useful for achieving them [13, 17]. We referred to the "Template Action Plan" (TAP) [40] that was prepared for our $\mathrm{HH}$ level when starting our initiative. However, once our $\mathrm{HH}$ initiative had started, we made our annual plans for the following year by choosing tools and activities mainly for the components that scored lowest in the HHSAF. This was effective not only because appropriate activities that were required at the moment were selected but also because it provided the frontline staff members convincing reasons why this particular activity was chosen for the year. By continuing this process, we were able to achieve high HHSAF scores for all five strategy components, which further increased the capacity for improvement. As we have reached the Advanced $\mathrm{HH}$ level, we are now working on the 'Leadership Criteria' of the HHSAF.

We found that ABHR consumption and HHSAF score were significantly positively correlated. We expected that both would increase as a result of the initiative but did not expect that the 2 variables would show such a strong correlation. A prior study from Japan [41] suggested that compliance would be improved by increasing the HHSAF score. In this study, the HH compliance rate obtained by direct observation and the HHSAF scores were compared between 3 Japanese hospitals; they ranked in the same order for both measurements. Our findings supported their results and indicated the possibility of adopting the HHSAF as a process measure in a single facility. This may be useful for some other hospitals as well, especially for those with low baseline compliance and HHSAF scores, when conducting a long-term initiative. Further reports from other hospitals and multicentre reports are needed to confirm this.

Our study was challenging in several aspects. Although national and subnational $\mathrm{HH}$ initiatives based on the WHO HH strategy have been introduced in many countries [3, 5-7], such full-scale initiatives had not been introduced in Japan by the time of this study, and the $\mathrm{HH}$ initiatives were left to each hospital's own efforts. In addition, having long-term care wards with many patients on ventilators, as well as daily recreational activities, made our situation even more complex. Furthermore, similar to many other nonteaching hospitals in our country, we could not afford additional personnel for covert observation or high-tech recording devices to assess $\mathrm{HH}$ compliance. However, by tracking the ABHR consumption, together with the HHSAF score as a process measure, we were able to complete our $\mathrm{HH}$ initiative successfully. Along with working on all five strategy components, repeating the review process at Step 5 of the step-wise approach for 5 consecutive years may have been one of the most important elements of our initiative. This has become a sustainable routine for us over this period of time, and we will continue repeating the cycles of the step-wise approach to sustain our improvement in $\mathrm{HH}$ practice.

There are several limitations in this study. First, this is a report from a single Japanese hospital, which provides long-term care for many patients with heavy medical needs and with no previous effective $\mathrm{HH}$ campaigns or initiatives. The amount of ABHR required in our hospital may be greater than that in many other hospitals that do not need to set such a high target. In addition, hospitals with higher $\mathrm{HH}$ compliance at baseline may not experience such an increase in ABHR consumption.

Second, we could not continuously record direct $\mathrm{HH}$ compliance. Some amount of ABHR may have been discarded or used incorrectly. As we did not provide incentives or punishment for the amount of ABHR consumed, we assume that there was not much advantage for each staff member to discard the substance. Although we found from our direct observations that the staff members with high $\mathrm{ABHR}$ consumption tended to use ABHR adequately, the possibility of discarded substance and incorrect use cannot be ruled out. In addition, the amount of ABHR that was used by patients and visitors was included in the ABHR consumption. As patient involvement in hand hygiene is recommended to improve the culture and climate of $\mathrm{HH}$ and to reduce hospital-acquired infections, we included patient/visitor ABHR consumption as part of the total $\mathrm{HH}$ improvement in our hospital.

Third, the amount we adopted as the adequate amount per $\mathrm{HH}$ event, $1.3 \mathrm{ml}$, is much less than the $3 \mathrm{ml}$ said to be recommended by most ABHR manufacturers, and even larger amounts were recommended for HCWs with large hands in a study from Europe [42]. The WHO guidelines recommend 20 to $30 \mathrm{~s}$ for each hand rub event, but some recent reports show that a 15-s application time is equal to a 30-s application in terms of wettability of hands [43] and is not inferior in terms of reducing bacterial counts on hands under experimental conditions [44]. Shortening the duration of $\mathrm{ABHR}$ application to $15 \mathrm{~s}$ may also improve compliance [45]. In our study, the $1.3 \mathrm{ml}$ ABHR that we used stayed wet for $20 \mathrm{~s}$ during the routine rubbing procedure for most participants, which may be because of the types of formula we used (mainly gel type, with moisturizing ingredients) $[20,21]$ and/or the fact that Japanese HCWs tend to have smaller hands than European HCWs. For HCWs with larger hands whose hands do not stay wet for at least $15 \mathrm{~s}$, we recommended 2 pushes $(2.6 \mathrm{ml})$ per $\mathrm{HH}$ event, but this proportion and amount was not analysed in this study.

Fourth, the outbreaks that we experienced within this study period-a two-drug-resistant Acinetobacter baumanii outbreak in 2014 and a multiple-drug-resistant Pseudomonas aeruginosa (MDRP) outbreak in 2016may have affected our results. Such outbreaks 
themselves can induce an increase in ABHR consumption, and the possibility of their influence cannot be excluded. However, the effects from these situations were expected to be temporary and limited to the ward in which the outbreak occurred. Our hospital-wide ABHR consumption continued to increase, regardless of the convergence of these outbreaks.

Fifth, the number of patient hospitalization days decreased between the pre-implementation period and the implementation period. This may be due to a change in the hospital policy in April 2014, which required a referral letter from every first visit patient. It is known that poor $\mathrm{HH}$ adherence is associated with higher patient-tostaff ratios [46], so the decrease in the number of patients may have had some influence on the increasing ABHR consumption per patient day. However, the $7.8 \%$ decrease in the mean number of patients alone could not have caused the eight-fold increase in the mean annual ABHR consumption (from 2013 to 2018), although it may have provided some positive effect.

Sixth, the HHSAF includes the amount of ABHR consumption as one of its scores. The maximum score given to the ABHR consumption is 5 points, which is $1 \%$ of the total score. $\mathrm{HH}$ compliance by direct observation is also included, with a maximum score of 30 points. Our score for direct $\mathrm{HH}$ compliance remained 20 points for the final 4 years. Altogether, our highest score for direct and indirect $\mathrm{HH}$ compliance was 25 points, which is $5 \%$ of the total score. This is not a large proportion; nevertheless, it cannot be said that the HHSAF score and ABHR consumption are completely independent variables.

\section{Conclusions}

We successfully implemented a WHO-based multimodal $\mathrm{HH}$ initiative in a nonteaching, secondary and long-term care hospital in Japan. Working on all five strategy components, repeating the cycle of the five steps of the stepwise approach for 5 years and assessing the HHSAF score at Steps $4 \& 5$ each year resulted in a continuous increase in ABHR consumption. Our results suggested that the HHSAF score may well be considered for adoption as a process measure within a single facility, although further investigation is necessary.

\section{Supplementary information}

Supplementary information accompanies this paper at https://doi.org/10. 1186/s13756-020-00732-7.

Additional file 1. Monthly alcohol-based hand rub consumption and patient hospitalization days.

\section{Abbreviations}

ABHR: Alcohol-based hand rub; FTE: Full time equivalent; GTI: Guide to Implementation; HCW: Health care worker; HH: Hand hygiene;

HHSAF: Hand Hygiene Self-Assessment Framework; ICC: Infection control committee; ICD: Infection control doctor; ICM: Infection control manager; ICN: Infection control nurse; ICT: Infection control team; ICU: Intensive care unit; LN: Link nurse; MDRP: Multiple-drug-resistant Pseudomonas aeruginosa; NHO: National Hospital Organization; PD: Patient day; TAP: Template Action Plan; WHO: World Health Organization

\section{Acknowledgements}

The authors acknowledge all the front-line staff members of NHO Shimoshizu National Hospital, who make personal commitments to care for patients with clean hands. The authors also thank the ICC, the ICMs, and the LNs for their continuous support and their leadership in leading local fieldbased initiatives.

\section{Authors' contributions}

MM and Y Suzuki defined the objectives of the study and led the study design and development. MM, SY and Y Suzuki were faculty members who developed and delivered the $\mathrm{HH}$ initiative. IM performed the data analysis and interpretation. Y Suzuki wrote the manuscript. All authors critically reviewed the manuscript and approved the final version.

\section{Funding}

Not applicable.

\section{Availability of data and materials}

All the data supporting the conclusions are available in the manuscript and in the supplementary file.

\section{Ethics approval and consent to participate}

Ethics approval was obtained from the NHO Shimoshizu National Hospital's Institutional Review Board for this study (authorization number 5, March 2017). Consent to participate was waived.

\section{Consent for publication}

Not applicable.

\section{Competing interests}

All listed authors have no conflicts of interest to disclose.

\section{Author details}

${ }^{1}$ Department of Pediatrics, National Hospital Organization (NHO) Shimoshizu National Hospital, 934-5 Shikawatashi, Yotsukaido 284-0003, Chiba, Japan. 2Division of Infection Control, NHO Shimoshizu National Hospital,

Yotsukaidou, Japan. ${ }^{3}$ Department of Nursing, NHO Shimoshizu National

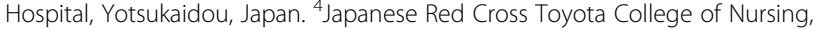
12-33 Nanamagari Hakusancho, Toyota 471-8565, Aichi, Japan.

Received: 29 January 2020 Accepted: 8 May 2020

Published online: 27 May 2020

\section{References}

1. Pittet D, Hugonnet S, Harbarth S, Mourouga P, Sauvan V, Touveneau S, et al. Members of the infection control Programme. Effectiveness of a hospitalwide programme to improve compliance with hand hygiene. Lancet. 2000; 356:1307-12.

2. Allegranzi B, Pittet D. Role of hand hygiene in healthcare-associated infection prevention. J Hosp Infect. 2009;73:305-15.

3. Stone SP, Fuller C, Savage J, et al. Evaluation of the national Cleanyourhands campaign to reduce Staphylococcus aureus bacteraemia and Clostridium difficile infection in hospitals in England and Wales by improved hand hygiene: four year, prospective, ecological, interrupted time series study. BMJ. 2012;344:e3005.

4. World Health Organization. WHO guidelines on hand hygiene in health care. Geneva: WHO; 2009. Available at: https://apps.who.int/iris/bitstream/ handle/10665/44102/9789241597906_eng.pdf (Last accessed January 2020).

5. Moro ML, Morsillo F, Nascetti S, Parenti M, Allegranzi B, Pompa MG, et al. Determinants of success and sustainability of the WHO multimodal hand hygiene promotion campaign, Italy, 2007-2008 and 2014. Euro Surveill. 2017;22(23).

6. Wetzker W, Walter J, Bunte-Schönberger K, Schwab F, Behnke M, Gastmeier $P$, et al. Hand rub consumption has almost doubled in 132 German hospitals over 9 years. Infect Control Hosp Epidemiol. 2017;38:870-2. 
7. Grayson ML, Stewardson AJ, Russo PL, Ryan KE, Olsen KL, Havers SM, et al. Effects of the Australian National Hand Hygiene Initiative after 8 years on infection control practices, health-care worker education, and clinical outcomes: a longitudinal study. Lancet Infect Dis. 2018;18:1269-77.

8. Sakihama T, Honda H, Saint S, Fowler KE, Shimizu T, Kamiya T, et al. Hand hygiene adherence among health care workers at Japanese hospitals: a multicenter observational study in Japan. J Patient Saf. 2016;12:11-7.

9. Buet A, Cohen B, Marine M, Scully F, Alper P, Simpser E, et al. Hand hygiene opportunities in pediatric extended care facilities. J Pediatr Nurs. 2013;28: 72-6.

10. Løyland B, Wilmont S, Cohen B, Larson E. Hand-hygiene practices and observed barriers in pediatric long-term care facilities in the New York metropolitan area. Int J Qual Health Care. 2016;28:74-80.

11. Murray MT, Cohen B, Neu N, Hutcheon G, Simpser E, Larson E, et al. Infection prevention and control practices in pediatric long-term care facilities. Am J Infect Control. 2014;42:1233-4

12. World Health Organization. Guide to implementation: a guide to the implementation of the WHO multimodal hand hygiene improvement strategy. Geneva: WHO; 2009. Available at: https://www.who.int/gpsc/5may/ tools/WHO_IER_PSP_2009.02_eng.pdf?ua=1 (Last accessed January 2020).

13. World Health Organization. Hand hygiene self-assessment framework 2010: introduction and user instructions. Geneva: WHO; 2010. Available at: https:// www.who.int/gpsc/country_work/hhsa_framework_October_2010.pdf?ua=1 (Last accessed January 2020).

14. Gould DJ, Moralejo D, Drey N, Chudleigh JH, Taljaard M. Interventions to improve hand hygiene compliance in patient care. Cochrane Database Syst Rev. 2017; (Issue 9):CD005186. https://doi.org/10.1002/14651858.CD005186.pub4.

15. Allegranzi B, Sax H, Bengaly L, Richet H, Minta DK, Chraiti MN, et al. Successful implementation of the World Health Organization hand hygiene improvement strategy in a referral hospital in Mali, Africa. Infect Control Hosp Epidemiol. 2010;31:133-41.

16. Chen JK, Wu KS, Lee SS, Lin HS, Tsai HC, Li CH, et al. Impact of implementation of the World Health Organization multimodal hand hygiene improvement strategy in a teaching hospital in Taiwan. Am J Infect Control. 2016:44:222-7.

17. Stewardson AJ, Allegranzi B, Perneger TV, Attar H, Pittet D. Testing the WHO hand hygiene self-assessment framework for usability and reliability. J Hosp Infect. 2013;83:30-5.

18. Kilpatrick C, Tartari E, Gayet-Ageron A, Storr J, Tomczyk S, Allegranzi B, et al. Global hand hygiene improvement progress: two surveys using the $\mathrm{WHO}$ hand hygiene self-assessment framework. J Hosp Infect. 2018;100:202-6.

19. Colombo C, Giger H, Grote J, Deplazesz C, Pletscherz W, Lüthi R, et al. Impact of teaching interventions on nurse compliance with hand disinfection. J Hosp Infect. 2002;51:60-72.

20. Macinga DR, Beausoleil CM, Campbell E, Mulberry G, Brady A, Edmonds SL, et al. Quest for a realistic in vivo test method for antimicrobial hand-rub agents: introduction of a low-volume hand contamination procedure. Appl Environ Microbiol. 2011;77:8588-94

21. Edmonds SL, Macinga DR, Mays-Suko P, Duley C, Rutter J, Jarvis WR, et al. Comparative efficacy of commercially available alcohol-based hand rubs and World Health Organization-recommended hand rubs: formulation matters. Am J Infect Control. 2012;40:521-5.

22. Al-Tawfiq JA, Abed MS, Al-Yami N. Promoting and sustaining a hospitalwide, multifaceted hand hygiene program resulted in significant reduction in health care-associated infections. Am J Infect Control. 2013;41:482-6.

23. Chun JY, Seo HK, Kim MK, Shin MJ, Kim SY, Kim M, et al. Impact of a hand hygiene campaign in a tertiary hospital in South Korea on the rate of hospital-onset methicillin-resistant Staphylococcus aureus bacteremia and economic evaluation of the campaign. Am J Infect Control. 2016; 44:1486-91.

24. Pittet D, Sax H, Hugonnet S, Harbarth S. Cost implications of successful hand hygiene promotion. Infect Control Hosp Epidemiol. 2004;25:264-6.

25. Larson E, Quiros D, Lin S. Dissemination of the CDC's hand hygiene guideline and impact on infection rates. Am J Infect Control. 2007;35: 666-75

26. Marra AR, Guastelli LR, de Araujo CM, dos Santos JL, Lamblet LC, Silva M Positive deviance: a new strategy for improving hand hygiene compliance. Infect Control Hosp Epidemiol. 2010;31:12-20.

27. Marra AR, Guastelli LR, de Araujo CM, dos Santos JL, Filho MA, Silva CV. Positive deviance: a program for sustained improvement in hand hygiene compliance. Am J Infect Control. 2011:39:1-5.
28. Mayer J, Mooney B, Gundlapalli A, Harbarth S, Stoddard GJ, Rubin MA. Dissemination and sustainability of a hospital-wide hand hygiene program emphasizing positive reinforcement. Infect Control Hosp Epidemiol. 2011;32:59-66.

29. Larson E, Murray M, Cohen B, Simpser E, Pavia M, Jackson O, et al. Behavioral interventions to reduce infections in pediatric long-term care facilities: the keep it clean for kids trial. Behav Med. 2018;44:141-50.

30. Jeanes A, Coen PG, Wilson AP, Drey NS, Gould DJ. Collecting the data but missing the point: validity of hand hygiene audit data. J Hosp Infect. 2015;90:156-62.

31. World Health Organization. Systematic literature review of automated/ electronic systems for hand hygiene monitoring preliminary results Geneva. Switzerland: WHO; 2013. Available at: https://www.who.int/gpsc/5may/ automated-hand-hygiene-monitoring.pdf (Last accessed April 2020).

32. Boyce JM. Electronic monitoring in combination with direct observation as a means to significantly improve hand hygiene compliance. Am J Infect Control. 2017:45:528-35.

33. Gould DJ, Creedon S, Jeanes A, Drey N, Chudleigh JH, Moralejo D. Impact of observing hand hygiene in practice and research: a methodological reconsideration. J Hosp Infect. 2017;95:169-74.

34. European Centre for Disease Prevention and Control. Point Prevalence Survey of Healthcare-Associated Infections and Antimicrobial Use in European Acute Care Hospitals. Stockholm: ECDC; 2013. Available from: https://ecdc.europa.eu/ sites/portal/files/media/en/publications/Publications/healthcare-associatedinfections-antimicrobial-use-PPS.pdf. (Last accessed June 2019).

35. Szabó R, Morvai J, Bellissimo-Rodrigues F, Pittet D. Use of hand hygiene agents as a surrogate marker of compliance in Hungarian long-term care facilities: first nationwide survey. Antimicrob Resist Infect Control. 2015;4:32-8.

36. Hansen S, Schwab F, Gastmeier P, PROHIBIT study group, Pittet D, Zingg W, et al. Provision and consumption of alcohol-based hand rubs in European hospitals. Clin Microbiol Infect. 2015;21:1047-51.

37. Kritsotakis El, Astrinaki E, Messaritaki A, Gikas A. Implementation of multimodal infection control and hand hygiene strategies in acute-care hospitals in Greece: a cross-sectional benchmarking survey. Am J Infect Control. 2018:46:1097-103.

38. Bert F, Giacomelli S, Ceresetti D, Zotti CM. World Health Organization framework: multimodal hand hygiene strategy in Piedmont (Italy) health care facilities. J Patient Saf. 2019;15:317-21.

39. Pfäfflin F, Tufa TB, Getachew M, Nigussie T, Schönfeld A, Häussinger $D$, et al. Implementation of the WHO multimodal Hand Hygiene Improvement Strategy in a University Hospital in Central Ethiopia. Antimicrob Resist Infect Control. 2017;6:3.

40. World Health Organization. Template action plan for WHO framework inadequate/basic results. Geneva: WHO; 2012. Available at: https://www. who.int/gpsc/5may/PSP_GPSC1_InadequateBasicWeb_Feb-2012.pdf?ua=1 (Last accessed January 2020).

41. Sakihama T, Kayauchi N, Kamiya T, Saint S, Fowler K, Ratz D, et al. Assessing sustainability of hand hygiene adherence 5 years after a contest-based intervention in 3 Japanese hospitals. Am J Infect Control. 2020;48:77-81.

42. Bellissimo-Rodrigues F, Soule H, Gayet-Ageron A, Martin P-Y, Pittet D. Should alcohol-based Handrub use be customized to healthcare Workers' hand size? Infect Control Hosp Epidemiol. 2016;37:219-21.

43. Paula H, Becker R, Assadian O, Heidecke CD, Kramer A. Wettability of hands during 15-second and 30-second handrub time intervals: a prospective, randomized crossover study. Am J Infect Control. 2018;46:1032-5.

44. Pires D, Soule H, Bellissimo-Rodrigues F, Gayet-Ageron A, Pittet D. Hand hygiene with alcohol-based hand rub: how long is long enough? Infect Control Hosp Epidemiol. 2017;38:1-6.

45. Kramer A, Pittet D, Klasinc R, Krebs S, Koburger T, Fusch C, et al. Shortening the application time of alcohol-based hand rubs to 15 seconds may improve the frequency of hand antisepsis actions in a neonatal intensive care unit. Infect Control Hosp Epidemiol. 2017;38:1430-4.

46. Kuzu N, Ozer F, Aydemir S, Yalcin AN, Zencir M. Compliance with hand hygiene and glove use in a university-affiliated hospital. Infect Control Hosp Epidemiol. 2005;26:312-5.

\section{Publisher's Note}

Springer Nature remains neutral with regard to jurisdictional claims in published maps and institutional affiliations. 